Cinémas

Revue d'études cinématographiques

Journal of Film Studies

\title{
Du cinéma à la carte
}

\section{Tom Conley}

Volume 10, numéro 2-3, printemps 2000

Cinéma et intermédialité

URI : https://id.erudit.org/iderudit/024816ar

DOI : https://doi.org/10.7202/024816ar

Aller au sommaire du numéro

\section{Éditeur(s)}

Cinémas

\section{ISSN}

1181-6945 (imprimé)

1705-6500 (numérique)

Découvrir la revue

\section{Citer cet article}

Conley, T. (2000). Du cinéma à la carte. Cinémas, 10(2-3), 65-84.

https://doi.org/10.7202/024816ar

\section{Résumé de l'article}

Dès qu'elle se voit à l'intérieur d'un film, la carte géographique, qui réfère donc au monde réel, trouble l'espace imaginaire de la diégèse tout autant que celui dans lequel une subjectivation spectatorielle a lieu. Une carte géographique, dans un film, fait signe vers un rapport de parties au(x) « Tout(s) » qui serait au fond de l'illusion réaliste que le film cherche à faire valoir. L'hypothèse est que la carte indique ce que Christian Jacob nomme la " bilocation du spectateur ". L'analyse porte sur l'espace parcellaire, tel que le travaille Gilles Deleuze au début de l'image-mouvement et sur le cinéma de Jean-Luc Godard, notamment une séquence tirée de Pierrot le fou, que l'on met en parallèle avec ses avatars passés (la fin de L'Enfer est à lui [R. Walsh]) et futurs (une séquence de La Haine [M. Kassovitz]). 


\section{Du cinéma à la carte}

\section{Tom Conley}

\section{RÉSUMÉ}

Dès qu'elle se voit à l'intérieur d'un film, la carte géographique, qui réfere donc au monde réel, trouble l'espace imaginaire de la diégèse tout autant que celui dans lequel une subjectivation spectatorielle a lieu. Une carte géographique, dans un film, fait signe vers un rapport de parties $\mathrm{au}(\mathrm{x})$ «Tout(s)» qui serait au fond de l'illusion réaliste que le film cherche à faire valoir. L'hypothèse est que la carte indique ce que Christian Jacob nomme la "bilocation du spectateur». L'analyse porte sur l'espace parcellaire, tel que le travaille Gilles Deleuze au début de L'Image-mouvement et sur le cinéma de Jean-Luc Godard, notamment une séquence tirée de Pierrot le fou, que l'on met en parallele avec ses avatars passés (la fin de L'Enfer est à lui [R. Walsh]) et futurs (une séquence de La Haine [M. Kassovitz]).

\section{ABSTRACT}

As soon as it it appears within a film, the geographical map, which referes to the real world, complicates the imaginary space of the diegesis as well as the space in which spectatorial subjectivication takes place. A map within a film hints at a relationship of the partials to the "Whole(s)," which would be basically the illusion of reality that the film seeks to exploit. The hypothesis is that the map points to what Christian Jacob has called the "bilocation of the spectator." This analysis focuses on fragmentary space, much like the work of Gille Deleuze in the beginning of The Movement Image, and on the cinema of Godard, and a sequence from Pierrot le fou in particular, viv-à-vis his avatars of the past (White Heat [R. Walsh]) and of the future (a sequence from La Haine [M. Kassovitz]). 
Dans le chapitre "Pointes du présent et nappes du passé » de L'Image-temps, Gilles Deleuze remarque que l'auteur de Hiroshima mon amour construit un film-cerveau, un monde articulé sur la mémoire, que s'approprie le spectateur. En réfléchissant sur la portée de l'œuvre d'Alain Resnais, peuplée selon lui d' "[...] un seul personnage, la Pensée» (1985, p. 159), Deleuze substitue aux longs et courts métrages du cinéaste le substantif carte. Chaque film serait une superposition de "nappes" de temps qui formeraient une carte. "Chaque carte en ce sens est un continuum mental, c'est-à-dire une nappe de passé qui fait correspondre une distribution des fonctions à une répartition des objets" (p. 159). La "méthode cartographique" consiste à additionner les cartes pour former un «diagramme», ou une «[...] superposition de cartes qui définit un ensemble de transformations de nappe en nappe, avec les redistributions de fonctions et les fragmentations d'objets" (p. 160). Ici comme ailleurs dans le texte de Deleuze, le lecteur est le témoin d'un passage fluide de nappes à cartes, et de l'ensemble de celles-ci au diagramme. Le "cinéma diagramme" de Resnais serait symptôme d'une vision philosophique et politique de plus grande envergure.

On trouve l'ancrage théorique de cette vision dans l'essai que Deleuze consacra à Foucault, dans un chapitre intitulé "Un nouveau cartographe» (1986, p. 31-51). Dans ce texte, carte et diagramme portent des inflexions historiques et politiques qui permettent de déplier le sens que Deleuze donne à nappe chez Resnais. L'histoire du monde moderne selon Foucault, dont Surveiller et punir décrit le système carcéral, dédouble celle de Resnais, qui apparaît dans la superposition virtuelle de Nuit et brouillard (1955), du Chant du styrène (1956) et de Toute la mémoire du monde (1958). Le réalisateur a le don de faire des diagrammes dès que les films-cartes sont laminés, superposés l'un sur l'autre et entreposés dans le souvenir du spectateur. Celui-ci pense aux sites et points de repère réels, établit des connexions: Auschwitz (Nuit et brouillard), c'est la Bibliothèque nationale rue de Richelieu (Toute la mémoire du monde), et les livres qui y sont "incarcérés" sont des corps-grains de plastique que les ouvriers transforment en objets néo-pétroliers (Chant du styrène), 
comme les cheveux et les poils de millions de cadavres étaient tissés en couvertures, elles-mêmes enroulées en cylindres. La force de ces images dans la pensée découlerait de la mobilisation de leur ensemble en tant que diagramme. L'archive concentrationnaire se métamorphose en ce que Deleuze va bientôt nommer un "devenir des forces». Une histoire du passé, telle l'histoire de la croissance de la prison et du corps carcéral comme "technique de l'âme» dans Surveiller et punir, vue et projetée dans le hic et nunc des luttes de notre époque, devient elle aussi diagramme (1986, p. 39-42). À l'intérieur de son cadre, toute carte du passé fait voir non la topographie limitée d'une société donnée, mais plutôt des points de repère différentiels, lieux où des énergies ou des actions pourraient se déclencher pour infléchir l'avenir. Un diagramme serait donc un ensemble de cartes qui suscite et focalise des travaux d'ordre critique et politique. Chez Foucault,

[...] [1]'histoire des formes, archive, est doublée d'un devenir des forces, diagramme. C'est que les forces apparaisserıt dans "toute relation d'un point à un autre" : un diagramme est une carte, ou plutôt une superposition de cartes. Et d'un diagramme à l'autre, de nouvelles cartes sont tirées. Aussi n'y a-t-il pas de diagramme qui ne comporte, à côté des points qu'il connecte, des points relativement libres ou déliés, points de créativité, de mutation, de résistance; et c'est d'eux, peut-être, qu'il faudra partir pour comprendre l'ensemble. C'est à partir des "luttes" de chaque époque, du style des luttes, qu'on peut comprendre la succession des diagrammes, ou leur ré-enchaînement par-dessus les discontinuités (p. 51).

Dans le cadre d'une étude cinématographique, il serait possible de reprendre les idées d'ouverture, de lutte, de style de cartographie, de style d'écriture, et de replier leur sens sur l'espace filmique. Car la carte cinématographique que propose Deleuze dans ses remarques sur les "nappes" de Resnais (le lecteur est tenté de lire "mappes", en songeant à l'écriture polyvoque de L'Imagetemps) implique, d'une part, que la lecture du film ait une dimension cartographique et que, d'autre part, le passage au diagramme, de l'archive à un devenir des forces, permette de passer 
d'une simple lecture à une lecture impliquée, engagée. Il s'agit d'instaurer une relation politique par le truchement d'une lecture créative. Cet acte trouverait sa source dans le principe, d'une simplicité aveuglante, qu'il existe un décalage entre l'ensemble d'un film et une carte, intériorisée par les images de la narration. Une "créativité » ou une «résistance» pourrait s'engager au lieu même où un tracé géographique ou topographique se trouve en miniature, là où, tout d'un coup, l'ensemble du film est perturbé par des cartes qui le mettent en abyme ou qui le déplacent de l'endroit où sa narration se déroule.

Une distinction s'impose. Deleuze construit le diagramme de l'ensemble des films d'un réalisateur-cartographe. Ici la proposition, plus modeste, ne porte guère sur le statut d'un auteur implicite qui concrétise sa vision du monde. Il s'agit plutôt, au moyen de la perception cartographique, de donner un style de lutte, de résistance, et même d'écriture au travail de lecture d'un film. Or, on constate qu'un film, qu'il s'agisse d'un court métrage ou d'un long métrage, trace une ligne de démarcation tantôt accidentée, tantôt tortueuse - entre le monde qu'il met en scène et le monde réel d'où il provient en un sens, mais dont il se détache aussi. Tout film se donne la tâche de construire une topographie des lieux, mais celle-ci n'a de sens que si elle altère les lieux et les espaces réels qu’enregistre la caméra.

$\mathrm{Vu}$ ainsi, le film serait un aide-mémoire de géographies plurielles et virtuelles. Mais il le serait à la manière d'un miroir brisé. Au fond, la lecture du film rappelle dans les espaces de sa projection une scission ou une faille. Dès le premier plan, le film obéit aux principes de la deixis qui situe le spectateur hors de l'écran où pourtant il s'imagine, se projette en regardant la bande-image. Ce que dit Christian Jacob à propos du regard lancé sur une mappemonde peut s'appliquer en effet à celui qu'on lance sur l'écran. L'un des pouvoirs les plus étranges du dispositif cartographique, dit-il, c'est "la bilocation du spectateur, qui est à la fois à l'extérieur de la carte, dans un lieu du monde réel, dans un environnement spatial familier, mais aussi à l'intérieur de l'image» (1992, p. 427-428). Ce qu'il appelle dissociation entre l'espace existentiel et vécu du regard et l'espace utopique de l'image vaut aussi pour le cinéma. Dans la ter- 
minologie deleuzienne, le spectateur devient «dividuel ", «fourchu", ou bien "bifurqué", autant par le média que par les actions qu'il présente (1986, p. 73).

Mais tout se complique lorsqu'une mappemonde, une carte ou un plan topographique se voit dans le champ de l'image. Il y a de nouvelles bifurcations dans les itinéraires cognitifs et mémoriels. La faille ouverte entre le spectateur et le film est élargie par la carte qui entraine de nouvelles lignes de faille: celle qui traverse le film et le sépare de sa véracité; celle qui traverse le spectateur et dissocie le bic et nunc de sa lecture de sa propre géographie intérieure, à savoir, sa subjectivité; celle qui soude et dissout à la fois les lieux et les espaces signalés par le paysage du film, ceux de la salle de projection et les lieux et espaces intérieurs du spectateur; celle qui construit dans le voir un fondu enchaîné de souvenirs historico-cinématographiques, parfois lézardés d'affects et de charges émotives, dans la mémoire du spectateur. Une fois qu'une carte géographique, petit aide-mémoire universel, s'insère dans l'image, le film trahit bien des médiations. La carte incarne l'espace du film, elle le miniaturise, tout en mettant en évidence le fait paradoxal que le film est là où il n'est pas. On retrouve alors la carte au plus profond de la division entre l'effet de réel du film et son statut de marchandise, ou encore de point de fuite sur l'horizon imaginaire d'un spectateur.

Limitons le champ de l'enquête en remarquant ce qu'opère la carte dans un petit nombre de genres et de styles filmiques. Dans le premier plan de Conte d'automne (Rohmer, 1998), le spectateur voit en écharpe, au bas de l'horizon et surplombant la ruelle d'un village méridional (attesté par les toitures en tuiles romaines), un panneau de signalisation routière traçant la carte des environs de Montélimar. Plus tard, la région est repérée grâce aux deux cheminées d'une centrale nucléaire. Ces cheminées constituent, dans l'espace filmique, un point de focalisation projective. Elles ressemblent au couple du récit. Figées dans le terroir, côte à côte, elles éternisent le désir de s'enraciner des partenaires. Dans les plans suivants, qui montrent en arrière-plan, au-delà des vignobles, le village et les réacteurs, le jeu de l'amour et du hasard se conjugue avec le paysage. 
C'est d'une tout autre manière que le nord de la France devient un des personnages principaux de La Vie de Jésus (Bruno Dumont, 1997). La ville de Lille et la campagne environnante du Pas-de-Calais semblent isolées et dépouillées, bien qu'elles se présentent sous un aspect riche et verdoyant. Le dénuement est tel qu'on a l'impression de se retrouver à la fin de la Première Guerre mondiale. Des plants de cultures fourragères et de blé poussent sur une mince couche de terre, parcourue par des panoramiques qui soulignent la ligne de démarcation entre le ciel et la platitude de l'horizon. Le paysage aplati s'implante dans l'histoire aussi par le truchement des émissions météorologiques et des nouvelles télévisées qui relatent les massacres d'Afrique Centrale: ces images, provenant du poste de télévision au-dessus du bar du bistro où se déroule une bonne partie de l'intrigue, sont autant de cartes qui signalent un monde off dont les turbulences présagent celles qui auront bientôt lieu in. À la fin d'India-Song (Duras, 1975), le non-lieu du salon où se déroule presque toute "l'action" du film est trahi et localisé par une carte de l'Inde. La caméra suit le tracé du Gange, concrétisant ainsi l'itinéraire de la mendiante dont la frêle chanson accuse, dès le premier plan du film, combien "le territoire de l'Ambassade ou du Palace [est] celui d'une Inde blanche vouée à l'extra-territorialité ", ou encore, combien l'Inde "est, par définition, négation" (Ropars-Wuilleumier, p. 206 et 208) ${ }^{1}$. La caméra suit les méandres du Gange, comme s'ils traçaient l'itinéraire de la mendiante dont le chant porte le nom du fleuve.

Le monde du film ne peut pas se situer sans allusion directe ou oblique soit au monde off, soit au monde dans lequel la projection se déroule, c'est-à-dire la topographie qu'il est censé représenter. Il faut une scission entre l'espace du monde et celui du film. Fuir là-bas, nous disent une bonne quantité de films fêtant les lieux de leur narration. C'est surtout le cas des films programmés dans les vols internationaux: les récits topographiques, qui sont le propre des longs métrages choisis par les lignes aériennes, font partie intégrante de leur économie. Les scénarios sont planifiés afin de donner aux spectateurs l'illusion de changer de lieu ou de culture dans les heures de somnolence passées à dix mille mètres d'altitude. L'Europe, c'est la cartographie des 
films vus à bord d'avions se dirigeant vers une ville européenne et l'Amérique, c'est le lieu de rêve offert dans les vols à destination de New York, Los Angeles, et autres villes américaines. Le monde du film serait l'endroit, anywhere out of this world, que cherche le spectateur afin de ponctuer ou de modifier le rapport qu'il entretient avec le monde environnant. On serait tenté de dire que la complexité du film, due aux conditions de sa réception et de sa production, est ainsi constituée par des nappes de géographies réelles et imaginaires, conscientes et inconscientes. Il arrive souvent que la cartographie concrétise et mobilise cette dimension. Là où, dans le film, se trouvent des représentations de l'espace, ce rapport est bien mis en évidence.

À cet égard, en abordant le cinéma du point de vue de la deixis, Christian Metz avait parlé d'une "géographie mobile» du film, dans laquelle il est impossible de fixer des positions d'énonciation et d'énoncé. Il dénonçait toute illusion de sens ou de message univoque dans le cinéma en raison de sa forme divisée: la bande-son scinde le lieu d'où est censé venir la bandeimage. Inversement, celle-ci renforce l'effet toujours flottant du son dans l'espace improbable figuré sur l'écran ${ }^{2}$. À la manière d'une écriture dans le champ de l'image, une représentation géographique peut signaler ou trahir tout un réseau de relations complexes entre le monde qui serait l'espace propre de l'imaginaire du film et les mondes dont le film se détache et avec lesquels il marque sa différence ou son décalage ${ }^{3}$.

Elle peut en même temps signaler des transactions opérées par le média cinématographique, sa rhétorique, avec le monde off. On peut cerner ici la négociation entreprise par le film en vue de ce qu'il ne peut qu'indiquer. Le monde devient son rival, l'objet qu'il doit écarter ou englober afin de constituer sa géographie propre et, pour ainsi dire, l'idéologie de son droit de cité. On peut observer ce travail cartographique dans Boudu sauvé des eaux (Renoir, 1932), un film qui ne perd rien de la fraîcheur de ses origines en bord de Seine. Ce n'est pas que la carte agisse comme plan de démarcation, suivant l'hypothèse d'un contraste entre le monde clos, sec, et molaire de l'adultère livresque dans l'appartement des Lestingois près du pont des Arts et les eaux qui coulent au-dessous, devant le Louvre, 
ouvrant le film sur des formes fugaces et moléculaires. Ce n'est pas non plus le fait que tous - personnages et spectateurs soient localisés ou déterminés par les cartes qui leur assignent une place et les invitent à rêver d'une échappée possible. Il s'agit plutôt de prendre en considération ceci : la carte souligne la profondeur du champ imaginaire du film même, notamment lorsque - après l'épisode de sa visite chez le coiffeur (en dehors de l'appartement, un peu ailleurs, un lieu signalé par les mots "service antiseptique" inscrits sur une vitre vue en contre-plongée) et la séduction d'Emma - Boudu fume un cigarillo dans le cadre dessiné par le pas de porte de la librairie.

On le voit déambuler entre le nom "Lestingois " peint sur la porte du magasin de son bienfaiteur à droite et, à gauche, deux cartes topographiques, vraisemblablement de la Belgique et de la mer du Nord, genre Sanson ou Blaeu de l'époque baroque, en vente derrière la vitrine. Dans un gros plan "oral" (et il y en a beaucoup), la lettre majuscule $S$ se trouve toute proche de la bouche de Boudu, de telle sorte qu'il paraît la fumer ou l'introjecter. Une topographie cocasse se met alors en jeu. Des régions là-bas, ailleurs, mais non des mondes totalisants de l'ordre des mappae mundi, se font remarquer. Dans les plans qui suivent, les cartes ne représentent rien qui nous guiderait ou qui préciserait la géographie parisienne tout au long du quai Malaquais. Pour le personnage sauvé des eaux mais perdu dans la circulation, ni l'effet localisant de la carte ni son efficacité schématique ne sont valorisés. La platitude de la carte est vite trahie par un plan d'ensemble, en contrechamp, qui pénètre l'espace du lieu.

Ici, le paradoxe d'un monde ou d'un tout à capter, qui se voit, mais reste morcelé ou insaisissable dans le champ de l'image, précise une thèse fondamentale de LImage-mouvement de Gilles Deleuze. Ce dernier suggère que le mouvement, sans lequel il n'y aurait aucune perception du temps ou de l'espace, se fait à partir d'un ensemble de singularités cernées par rapport à un Tout :

[O]n se donne un Tout, on suppose que "tout est donné", tandis que le mouvement ne se fait que si le tout n'est ni donné ni donnable. Dès qu'on se donne le tout dans l'ordre éternel des formes et des poses, ou 
dans l'ensemble des instants quelconques, alors ou bien le temps n'est plus que l'image de l'éternité, ou bien la conséquence de l'ensemble (1983, p. 17).

D'où l'observation que le mouvement est une "translation dans l'espace", rendue manifeste par un déplacement de parties dans l'espace ainsi qu'un "changement qualitatif dans un tout " (1983, p. 18), de sorte que, "quand Achille dépasse la tortue, ce qui change, c'est l'état du tout qui comprenait la tortue, Achille, et la distance entre les deux» (p. 18). Ou encore, tout mouvement se comprend par "un mouvement de migration ou une variation saisonnière" (p. 18). L'exemple que Deleuze tire de Bergson pour illustrer le mouvement de translation semble familier. Il note d'ailleurs qu'il est si célèbre "que nous ne savons plus voir ce qu'il a de surprenant» (p. 19). Lorsqu'on met du sucre dans un verre d'eau, on doit attendre que le sucre fonde, mais à l'aide d'une cuiller la personne "peut hâter cette dissolution" (p. 19). L'action de l'ustensile précipite la transformation de l'ordre d'Achille, de la tortue, et de la distance entre la bête et l'homme.

Cet exemple banal et quotidien ne nous rappelle-t-il pas également une séquence de Deux ou trois choses que je sais d'elle (Godard, 1966), dans laquelle un homme se met à philosopher sur les espaces du monde ou encore cherche à cerner les rudiments de la cosmographie? Un jeune homme, penché au-dessus de la tasse de café qu'il contemple, après avoir mis une cuillerée de sucre dans la masse brune, étudie l'effet enivrant des galaxies, ici des agglomérats de bulles qui remuent, tournent et se dispersent dans le courant créé par la cuiller. "Si j’agite [...]", commence Deleuze en se souvenant, semble-t-il, de cette séquence pascalienne (mimant les «deux infinis » des Pensées) du long métrage de Godard: "Si j'agite avec la cuiller, j'accélère le mouvement, mais je change aussi le tout qui comprend maintenant la cuiller, et le mouvement accéléré continue d'exprimer le changement du tout» (1983, p. 19). Deleuze finit par définir le tout par le terme relation, «extériorité ouverte». De là découle la distinction du tout et des "touts" d'avec les ensembles, mondes clos ou artificiellement déterminés. Ceux-ci sont plutôt clos et ceuxlà plutôt ouverts. On dirait que le verre d'eau ou de café est clos 
mais s'ouvre lorsque «le tout se crée, et ne cesse de se créer dans une autre dimension sans parties, comme ce qui entraîne l'ensemble d'un état qualitatif à un autre, comme le pur devenir sans arrêt qui passe par ces états" (p. 21).

L'effet exemplifié par la tasse de café, avant que Deleuze ne l'explicite au départ de L'Image-mouvement, renvoie à un rapport quasiment classique qui distingue la cosmographie de la topographie $^{4}$. Celle-là traite du tout et de l'univers dans la mesure où l'on connaît les parties que l'on peut observer et nommer, tandis que celle-ci se consacre aux détails, aux parties, aux lopins du monde ${ }^{5}$. L'analogie qu'a illustrée Pieter Apian, dans ses éditions latines et vernaculaires de sa Cosmographia, a lancé une image excédant le discours de l'équation reçue : la représentation du monde (de la terre et ses continents, ses océans, ses mers et ses dénivellations de masses) se compare au portrait d'un homme barbu, comme un plan de ville se compare au dessin d'une oreille ou d'un œil, alors même que ceux-ci sont des organes sensibles qui enregistrent à la fois la similitude et l'aspect de la ville et du monde. Dans la légende, l'acte de la perception même est supprimé de l'analogie. L'homme qui allégorise "la peinture » - et qui ressemble au portrait du Christ - a le monde (comme cosmographie) devant les yeux. Son point de vue est orienté vers le "tout" de la sphère terrestre. Il y a dans la similitude d'Apian une sorte d'image-perception, dispositif qui fête la naissance de la visibilité de ce qui, avant la mission Apollo en 1969, était entièrement invisible. Le portrait de l'homme serait l'image-affection ou la visagéité même, le mouvement de traduction de l'émotion dans la physionomie d'une relation de deux ensembles ouverts, c'est-à-dire l'homme et le monde circonvoisin.

Dans le très gros plan de Deux ou trois choses que je sais d'elle, les "touts" sont en branle. La tempête brownienne dans une tasse de café signale que Godard, cosmographe en quelque sorte en 1966 - et l'année précédente avec son allusion aux cosmonautes américains dans Pierrot le fou (1965) - anticipait l'avènement de l'image photographique du globe terrestre vu de la nacelle du satellite. À cet égard, les années 1968-1969 étaient des années ptoléméennes par excellence, les plus métaphysiques 
et concrètement "topographiques" aussi — surtout dans le sens de la géographie politique. Dans les termes d'une cosmographie où le monde est traité comme sphère terrestre, Godard soulignait à la fois dans Deux ou trois choses que je sais d'elle et dans Pierrot le fou que la relation entre parties (êtres humains) et touts (la nature, le monde entendu comme biosphère) est lacunaire et sans compossibilité (dans le sens leibnizien) ${ }^{6}$. Comme l'a crié Ferdinand dans un plan d'ensemble de Pierrot le fou, alors qu'on voit un bateau à moteur s'éloigner du port de Toulon vers le large méditerranéen, "je ne suis qu'un point d'interrogation sur l'horizon » de l'univers.

Sans doute Godard visait-il une tératologie qui fait partie de la tradition carto-cosmographique de la réforme des humanistes du XVI ${ }^{e}$ siècle, portés eux-mêmes à penser aux différentes fins du monde et aux derniers jugements, du moins dans le cadre d'une géographie métaphysique. Les points qui rattachent les réformateurs protestants de Genève, en gros des géographes, à l'œuvre de Godard sont nombreux. C'est ce que remarque le lecteur à la fin des Simulacres de la mort (1538) de Hans Holbein? ${ }^{7}$ Dans une gravure sur bois, Holbein dépeint une relation cosmographique des plus problématiques. Dans le champ minuscule de l'image de bois $(4,76 \mathrm{~cm}$ sur $6,35 \mathrm{~cm})$, une femme nue porte la main au ciel, vers le créateur (barbu, un peu renfrogné, et apparemment fatigué du travail en série), ses doigts touchent à la terre sur l'axe d'une sphère armillaire. L'image a ceci de paradoxal que la terre, caressée par la femme et dessinée selon un ordre aristotélicien (comme dans le frontispice de la Protomathesis d'Oronce Finé, six ans avant la parution des Simulacres), n'est pas un monde, mais une représentation topographique, voire un paysage. Le monde, qu'on aurait dû imaginer d'un point de vue impossible (du moins avant 1968), est tout d'un coup pensable dans les parties constituantes, les détails ramassés, d'une topographie vallonnée où figurent trois collines superposées, un peu accidentées par des haies ou des touffes de végétation, une maison çà et là et le tout sous le siège de Dieu à la fin de l'ère humaine.

Il est clair que Godard jouait sur les fins possibles de l'homme dans le dénouement de Deux ou trois choses que je sais d'elle. 
L'extinction d'une cigarette, vue en très gros plan, signifiait la fin d'un univers météore. Le plan est en dialogue avec le silence qui allie la mer au soleil après le suicide de Pierrot. C'est la même connotation que nous trouvons dans le titre bilingue de Week-End, où il est question d'une fin médiocre, celle de la dissolution d'un monde débile ravagé par le genre humain. Il se peut que Godard ait eu son mot à dire sur l'apocalypse brassée par l'industrie américaine afin d'inaugurer des effets chaudsfroids au début de la Guerre froide, à l'ère nucléaire : la conclusion de Week-End et celle d'autres films parus entre 1964 et 1968 font allusion à la cosmographie et à la paranoïa américaines de l'après-guerre. Dans White Heat (L'enfer est à lui, Raoul Walsh, 1949), une tératologie est simulée par un paysage de hortonsphères où règne Cody Jarret, quelques instants avant son auto-immolation et celle de la planète - «Top 'o the world, $\mathrm{Ma}$ !". Cody Jarret se met à tirer au hasard et finit par se faire exploser. L'événement qui couronne L'enfer est à lui était une sorte de pressentiment du cinéma "flamboyant» de Godard.

Il suffit de revenir à Pierrot le fou, à la séquence du vol et du guet-apens de Marianne. Elle descend ses adversaires au moyen d'une carabine montée d'une loupe, en hommage peut-être aux engins des tireurs de High Sierra (Raoul Walsh, 1941) et de L'enfer est à lui. Quand elle vise sa proie qui se trouve prise au piège dans un filet, l'iris où apparaissent, du point de vue monoculaire de Marianne qui regarde par la loupe, les hommes qu'elle cible, est en même temps un monde total, un tout à la fois dans et n'ayant rien à faire avec l'espace circonvoisin. Liris reproduit le schéma d'un monde à la mode de l'orbis terrarum. Dans le contexte de Pierrot le fou, où tout objet est autre qu'il n'est en réalité, on se souvient du support sur lequel est placée la Ford «Galaxy ", la voiture que Pierrot et Marianne vont finir par voler. Le support est un appareil hydraulique servant pour le graissage et la vidange, mais c'est aussi une base qui fait de la voiture une sculpture industrielle avant même ou sans qu'elle ne subisse un travail d'artiste. Or dans la séquence du guet-apens, il se trouve que l'iris est une mappa mundi qui correspond - sans correspondre - aux planètes et aux étoiles qui criblent le film. C'est un plan-emblème du "monde" que tient la femme entre ses 
mains dans une des séquences suivantes qui se passe à l'intérieur d'une salle de bowling: le globe noir de la boule qu'elle tient serait le pendant de la forme dessinée par l'iris encadrant et figurant une mappa mundi médiévale.

Or, on pourrait dire que le monde redevient le sujet du cinéma de la décennie 1990-2000 qui marque ses affinités avec la nouvelle vague et son legs. Matthieu Kassovitz retourne à Pierrot le fou et à L'enfer est à lui au début de La Haine (1995), à l'exception près que le monde est moins un objet inscrutable qu'une figure allégorique qui fige ce qui, chez Walsh et Godard, était de l'ordre de l'implicite ou du sous-entendu. Dans La Haine, le seul plan en couleurs est celui du monde vu de la mission Apollo. Précédant le générique, le monde spatialise la durée, intolérable, de la conscience d'un monde qui vit ses derniers instants. La bande-son raconte l'histoire d'une personne qui se jette par la fenêtre de l'immeuble qui la retenait prisonnière. Le corps qui tombe éprouve le plaisir de suspension dans le laps de temps qui sépare le début de sa chute de son écrasement. Lécrasement est simulé sur la bande-image par un cocktail Molotov, qui descend des espaces infinis, comme un météore avant de venir frapper, en l'espace d'une demi-seconde, la planète et de la faire éclater en morceaux. Une sorte de big bang, ou implosion de fin du monde, se voit dès le premier plan: dans l'allégorie du film, l'avantgénérique semble anticiper sur le petit bang du revolver de Vinz qui mène le drame à sa fin tragique ${ }^{8}$. La voix off d'Hubert en dramatise l'impact: "C'est l'histoire d'une société qui tombe et qui au fur et à mesure de sa chute se répète sans cesse pour se rassurer, jusqu'ici tout va bien, jusqu'ici tout va bien, jusqu'ici tout va bien, l'important ce n'est pas la chute, c'est l'atterrissage .". Dans cette répétition de l'énoncé (qui va revenir encore deux fois dans le film), le mot "atterrissage " est prononcé à l'instant où explose le monde ${ }^{10}$. L'accélération de la durée que l'on observe dans cette juxtaposition du temps de la narration (une question de deux ou trois secondes) et de la perpétuité de la Terre dans le système solaire (là où le temps n'est ni successif ni simultané) accuse le mécanisme médiatique du destin. C'est le dispositif déployé dans les intertitres, empruntés à la rhétorique visuelle de $60 \mathrm{Mi}$ nutes et d'autres émissions télévisuelles d'actualités, qui fait que 
La Haine rend explicite le pouvoir que la télévision du dimanche croit exercer sur le destin du monde.

À deux reprises ce titre-emblème revient dans la diégèse. Les trois jeunes héros passent deux fois devant une affiche publicitaire qui montre la même image, vue en blanc et noir, au-dessus de la légende qui annonce: "Le monde est à vous." Ainsi que le décrit la séquence 65 du scénario:

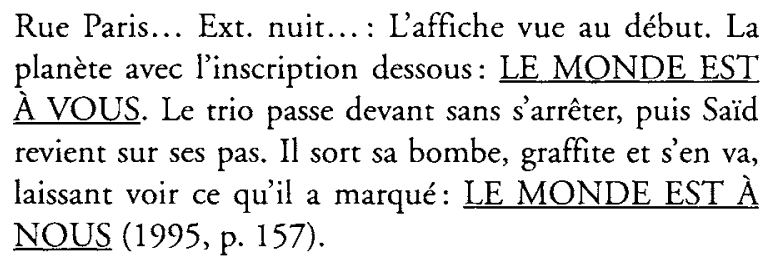

Sur le plan de la propagande touristique, la bombe de peinture équivaut au cocktail Molotov. N'importe quel endroit du monde, indique la publicité, est à la portée du bras du voyageur terrestre bien aisé ${ }^{11}$.

Mais dans l'univers ciné-cartographique, il est fait une allusion directe à la même formule de Scarface (Howard Hawks, 1932), où une affiche porte le slogan "The world is yours". L'ambition galopante du criminel-protagoniste (Paul Muni) est soulignée par l'impossible dénoté dans l'affiche qui se trouve au fond de plusieurs plans d'ensemble. Ici, il est impossible d'écarter la référence sous-jacente à deux autres films de la même époque. La vie est à nous (Jean Renoir, 1934) traite de la vie quotidienne des exclus et des victimes de la Grande Dépression. La vie est à nous partagerait avec La Haine la même dimension politique, à la différence près que là où La vie est à nous projette sur un plan collectif la vision d'un avenir collectif, Renoir voyant une issue dans le socialisme du Front populaire, le film de Kassovitz induit quant à lui de l'amertume ou encore le sentiment de la futilité des choses devant un monde marqué par le chômage et les conflits sociaux. L'écho du Ciel est à vous (Grémillon, 1944) se fait aussi entendre. Dans ce film qui traite de l'avenir de la France sous l'occupation, les citoyens d'un village français rural (où le maréchal Pétain est comme l'avatar du Front national et de l'idéologie des skinheads) regardent dans le ciel strié la maté- 
rialisation de leur désir de voler. Le rêve de planer au-dessus de la terre va contre l'immobilité de ce bas monde. Or cette immobilité est repliée dans la référence implicite à Grémillon. Dans les trois films, une cartographie est marquée dans la différence du "monde" ou de la "vie" avec l'habitant de la terre. Il est impossible d'effectuer le vol d'Icare qui permettra à l'individu de gagner un point de vue qui serait au-dessus de la terre. Cette contradiction travaille la politique de chaque film de façon variée, tantôt par une vague d'utopisme, tantôt par une ironie grinçante, tantót par un camouflage équivoque.

Dans La Haine, le retour de l'affiche publicitaire est lié à la topographie présentée dans une séquence qui le précède immédiatement. Signalé par l'intertitre " 2: 57 a.m." dans la version américaine, il s'agit du moment où les trois jeunes hommes pensent à leur destin après avoir manqué le dernier métro pour la banlieue. Ils regardent le paysage de la ville du toit d'un bâtiment. La tour Eiffel est située au point de fuite du plan d'ensemble. Hubert raconte l'histoire de l'homme qui tombe de l'immeuble de cinquante étages. Dans un plan moyen, entre la tête d'Hubert et celle de Vinz (vues de dos), les lumières de la tour - emblème de la ville — vont bientôt s'éteindre et disparaître dans la nuit, progressivement, de haut en bas. La "chute » de la lumière a l'effet d'entériner celle de l'histoire d'Hubert. La rotondité du crâne de Vinz suggère un monde proche qui est la contrepartie du paysage au loin. Le globe-tête met en jeu la ressemblance de Vinz avec les skinheads qu'il déteste, soit aussi celle du juif (qu'il est) avec ses mortels ennemis. Force est de constater que la contradiction la plus surprenante du plan se trouve dans le jeu de distances, entre un "monde" proche et un paysage portant l'emblème du pays au loin. La tête de Vinz est donc une espèce d'analogon $\mathrm{du}$ "monde" vu dans le générique et dans l'affiche du plan suivant. Elle lie les deux plans en même temps que sur la bande sonore se répète la même histoire de chute.

Dans le dialogue, Saïd, spécialiste ès graffiti et chroniqueur pour qui la bombe remplace la plume, offre un morceau de poésie à un Vinz perdu dans une pensée qui ressemble aux deux infinis de Pascal. Il lui dit: "Voici un poème", ce que le sous-titre anglais traduit par «Check out this shit»: 
Le pénis de Le Pen

À peine il se hisse

Hubert ajoute: «Si Dieu te fait chier, c'est lui qui fait pousser le caca." On en déduit donc que Dieu habite les boyaux de Vinz. La dimension métaphysique du film, à vrai dire le propre de sa cosmographie, retourne un peu comme le refoulé, à la séquence inaugurée par l'intertitre indiquant qu'elle a lieu à 5 h 04 . Ici le globe terrestre de l'affiche "Le monde est à vous" se voit audessus des trois promeneurs. Saïd macule le $V$ de vous et inscrit au-dessus un $N$. Un gros plan insiste sur l'effet de différence (si le sous-titrage équivaut à une interprétation de ce qu'il traduit, le spectateur anglophone verra pour une deuxième fois, mais ici en caractères gras, la formule tirée de Scarface).

Il y va de la chute des personnages et du monde dans lequel, perdus, ils circulent. Mais la lettre $N$ dirige le sens du film un peu ailleurs. La lettre transcrit le vocable du titre $-\mathrm{N}=$ haine - tout en laissant tomber l'article qui définirait l'allégorie du titre, dans la mesure où le film aurait comme sujet "la" haine en général. Avec l'affaissement du sens, effet de la marque de la lettre $N$ qui instaure une "cacographie» dans l'écriture de l'affiche, il est licite de lire dans les espacements du texte «le monde est à nous" les questions suivantes: Est-il à nous? À $n$ où? Le sens du film bascule entre nous et vous, entre $v$ et $n$, entre deux lettres, l'une imprimée et officielle, l'autre greffée ou plutôt barbouillée au-dessus. Dans la perspective de l'histoire de cette expression, le jeu signale que l'avenir tel qu'il est perçu dans le film des années trente est passé et mis à $n u$ ici, sans prétention politique mais tout en se situant dans l'économie restreinte d'un réseau d'allusions aux films classiques et proto-noirs de l'entredeux-guerres, d'autant plus si l'on pense au travail de la lettre dans $M$ le Maudit de Fritz Lang ${ }^{12}$. Le graffiti ouvre sur un univers d'affiliations si enchevêtrées que l'on pourrait dire avec Gilles Deleuze que "l'image est lisible autant que visible" (1983, p. 24).

Dans la séquence 28 de La Haine, Vinz traverse en plein jour la cour de l'ensemble des HLM où habitent ses parents et les familles de ses copains. Tout à coup, il aperçoit une vache holstein qui traverse la cour extérieure. Ainsi que l'indique le scénario: 
En bas, Vinz et Said marchent dans la rue, eux aussi écoutent la musique, les yeux levés pour essayer d'identifier d'où vient le son. Vinz baisse les yeux. Devant lui, à une vingtaine de mètres, une vache blanche passe avec dédain pour disparaître derrière un immeuble (1995, p. 88).

Vinz s'exclame: "Téma!", et par là même, il semble mettre le doigt sur le thème cartographique du film. Saïd veut savoir ce que son ami vient de voir, et Vinz lui répond: "La vache, elle était là!» La remarque lui attire la raillerie de Saïd: "Ta gueule avec ta vache... C'est toi, la vache» (p. 88).

S'agit-il d'une vision de type melvillien, un peu comme celle de la baleine blanche à l'horizon de Moby Dick? De l'irruption du sacré dans le quotidien dégradé? Ironie d'un bovin, autrefois littéraire si l'on pense aux romans de Flaubert, qui traverse le plan d'un lieu quelconque? Est-ce un jeu de mots qui joue sur l'image de la vache et l'exclamation de Vinz - «Téma!" - en lieu et place d'un banal mais plus attendu "Ah! la vache!", qui conduit le spectateur à croire que Vinz voit une carte du monde sur la peau de la vache? La vision est construite un peu à la manière de l'œuvre de Claudio Parmigginai, Tavole zoogeografiche (1968, date à peu près synchrone de l'image au générique), qui substitue aux taches noires sur fond blanc de la peau de vache les cinq continents du monde ${ }^{13}$. Vinz est fou, mais il aussi clairvoyant que le spectateur du générique qui voit le monde d'un point de vue lunaire. Sa vision double la cosmographie vue au début de $L a$ Haine. Or, Vinz devient cartographe en fusionnant, à l'instar de Deleuze au début de LImage-mouvement, fragments et totalité, lopins du monde et le monde même. Le mélange de mers et de continents qu'il voit sur la peau de vache constitue, comme le remarque Saïd, la vision d'une personne au bord de la psychose.

Sur un plan thématique maintenant, la vache n'a pas de lieu au lieu où elle se promène. Comme les personnages qui chôment, et qui le deviennent à leur manière, elle figure la bête dans une jungle de béton armé. Avec elle se rappelle à notre bon souvenir le paysage où broutent les bovins, mais aussi le souvenir d'un espace off dans lequel, ailleurs, les humains s'entre-parasitent. Le rapport entre la partie, ici figurée par l'image d'un tout, et un grand 
ensemble ne saurait être plus clair. "À chacun son métier et les vaches seront bien gardées», nous rappelle Vinz (Kassovitz, p. 156), indiquant que la vache qui chôme n'est guère la vache qui rit. Le globe terrestre inaugurant la réflexion sur l'espace lacunaire et fragmentaire de la banlieue ouvre le film aux conflits de classes, de générations, et de races que la métaphysique et la cosmographie pascaliennes ne sauraient ni apprivoiser ni résoudre. La cartographie du film mobilise sa politique tout en restant, comme le réseau de ses allusions aux cinémas de l'entre-deux-guerres et de la nouvelle vague le montre, dans une tradition classique.

En guise de conclusion, disons que dans chaque film cité, de Boudu sauvé des eaux jusqu'à La Haine, la force de ce que Deleuze appelle la Pensée est travaillée par les enjeux de sa cartographie. La carte dans le film, ainsi que la carte comme ensemble de films, fait partie de sa fonction diagrammatique. La carte est en quelque sorte le lieu où commence ce que Deleuze avait appelé le devenir des forces du cinéma. Il reste à préciser et à développer une politique de lecture et d'interprétation à partir de ces données et de ces premières incursions.

\section{Texte français établi avec le concours de Marion Froger}

Harvard University

\section{NOTES}

1 Pour une lecture cartographique du film hollywoodien, voir mes Film Hieroglyphs: Ruptures in Classical Cinema (Minneapolis: University of Minnesota Press, 1991, p. xxvi et 87-90).

2 Pour Metz, cette géographique mobile va à l'encontre d'un échange où il existe un énonciateur et un "énonciataire" et où il y a de la "communication", le jeu du cinéma offrant " $[\ldots]$ des directions intérieures à la géographie du film, des orientations" que le travail de l'analyse doit déceler (L'énonciation impersonnelle ou le site du film, Paris: Méridiens Klincksieck, 1991, p. 34-35). Metz trahit un mouvement qui voudrait stabiliser le parcours d'une déstabilisation. Cela se remarque dans le va-etvient de ses propos, là où le je est en jeu: "Je vais donc regagner le terrain... " en revenant aux textes de base (p. 35); "Je vais donc parcourir le champ de ces figures d'énonciation [...], la géographie changeante des angles sous lesquels le texte se présente à son spectateur... Litinéraire que j'ai choisi me fera visiter [...] une petite centaine de sites énonciatifs." (p. 35-36).

3 Il serait fécond - mais ici ce n'est pas le lieu - de rattacher la deixis dans le cinéma à ce que Francis Wolff appelle le "rapport du dire au monde" (Dire le monde, 
Paris: P.U.F., 1997). La littérature, dit-il, «[...] plus qu'aucune autre expérience de parole $[. .$.$] nous donne l'image de cette totalité infinie qu'est la parole-monde. Elle$ représente l'espace où se réalise tous ses possibles", où se fait "[...] l'expérience partielle de ce tout " qu'est le monde (p. 180). De sorte que dire, c'est instaurer une espèce de cartographie de et par la parole.

4 Voir la première phrase du premier livre de la Geographia de Ptolémée (autour de 145 A.D.)

5 Lucia Nuti analyse finement et de manière détaillée la fortune de l'axiome de Ptolémée, surtout pour ce qui concerne la peinture et la topographie, dans "Le langage de la peinture dans la cartographie topographique" (Catherine BousquetBressolier (direction), L'CEil du cartographe et la représentation géographique du Moyen Age à nos jours, Paris: CHTS, 1995, p. 54).

6 À ce sujet, voir le chapitre 3 du Pli. Leibniz et le baroque de Gilles Deleuze (Paris: Minuit, 1988).

7 Voir The Dance of Death: 41 Woodcuts by Hans Holbein the Younger (Werner Gundersheimer (direction), New York: Dover, 1971, p. 55).

8 Le policier du toit (vu depuis la séquence 11, qui se passe sur le toit d'une HLM dans la banlieue où résident les trois protagonistes) tire sur Vinz après avoir crié: « Regardez comme il chie dans son $f . . . "$. Le texte est celui du scénario avant tournage, publié sous le titre Jusqu'ici tout va bien... Scénario et photographie autour du film La Haine (Mathieu Kassowitz, Montpellier: Actes Sud, 1995, p. 181); on notera que les dialogues du film sont encore plus crus que ceux de la version imprimée.

9 Il semble y avoir trois versions du commencement. Dans le texte du scénario avant tournage, on trouve: "C'est l'histoire d'un homme qui tombe d'un building de cinquante étages. À chaque étage, au fur et à mesure de sa chute, il se répète [...]" (Mathieu Kassowitz, Jusqu'ici tout va bien... Scénario et photographie autour du film $\mathrm{La}$ Haine, Montpellier: Actes Sud, 1995, p. 17). Alors que la version française du film donne: "C'est l'histoire d'un homme qui tombe d'un immeuble de cinquante étages. Le mec, au fur et à mesure de sa chute, il se répète [...]" (transcription de la version par Jenny Lefcourt, que je tiens à remercier ici). Pour le public international, c'est la société qui choit ; et pour les auditeurs français ou francophones, il s'agit d'un homme qui sera, dans la version ultime, marqué en tant que mec qui tombe non pas d'un immeuble mais d'un building de 50 étages. Chaque version présente une symbolisation et produit un effet particuliers.

10 Il faut noter encore que, dans le scénario, la phrase " [...] mais l'important, ce n'est pas la chute, c'est l'atterrissage" est absente. Le scénario insiste sur le fait que l'image du monde est une affiche, "une photographie collée sur un panneau publicitaire" qui s'enflamme au toucher du cocktail Molotov. Dans la version française, "c'est l'atterrissage" (voix off) accompagne l'image de l'explosion. Les modifications apportées au générique portent à croire quil a fait l'objet d'une attention toute particulière; les raisons qui les ont motivées restent à expliquer.

11 Il s'agit là de la première allusion explicite à l'affiche. À la différence du scénario écrit qui ne l'indique pas, l'affiche se voit de façon fugace lorsque Sam, Saïd et Vinz roulent en voiture (Mathieu Kassowitz, Jusquici tout va bien... Scénario et photographie autour du film La Haine, Montpellier: Actes Sud, 1995, p. 57).

12 Le film s'éloigne, et de beaucoup, de la version écrite de la séquence. Dans le texte, le trio regarde Paris depuis les toits. Un gros plan capte «[...] un paquet de feuilles à rouler $\mathrm{OCB}$. Vinz finit de rouler un joint et le passe à Hubert qui l'allume" (Jusquici tout va bien... Scénario et photograpbie autour du film La Haine, Montpellier: Actes Sud, 1995, p. 155). Hubert et Vinz regardent les étoiles pendant que Saìd «[...] est en train de taguer les cheminées avec sa bombe récupérée ". La métaphysique de la 
séquence est trahie par la réplique de Vinz qui dit, le regard porté sur les étoiles: «On se sent peu de chose, pas vrai? " (p. 156). Hubert s'en prend à Vinz en raison de de son air philosophique: "Tu en as d'autres, des phrases à la con comme ça?" Vinz répond en citant des proverbes qui masquent ou qui corrigent son penchant pour la métaphysique: "Petit-à-petit l'oiseau fait son nid "; "À chacun son métier, les vaches seront bien gardées"; "J'en ai des tas comme ça. " Pour ceux qui s'intéressent aux sources et variations de La Haine, deux éléments ressortent des écarts entre le texte et le film. Premièrement, la cosmographie et l'aspect "pensée » sont rendus par les toits de la ville, mais sans l'icône de la tour Eiffel, remplacée par la formule «Liberté, égalité, fraternité " énoncée par Hubert. Dans la version écrite, avant la récitation de l'histoire de la chute de l'immeuble, le dialogue véhicule la contradiction. En second lieu, l'allusion aux "vaches bien gardées" est reliée, comme nous allons le voir, à la vision "cartographique" de Vinz.

13 La vache en tant que carte est un topos, voire un thème, qui rattache la science de la mensuration du monde à la fantaisie de l'artiste. Voir à ce sujet l'illustration de la vache de Parmiggiani et l'analyse de ses éléments dans l'ouvrage de Christian Jacob intitulé L'Empire des cartes. Approche théorique de la cartographie à travers l'histoire (Paris: Albin Michel, 1992, p. 385-387 et illustration 39).

\section{OUVRAGES CITÉS}

Conley, Tom. Film Hieroglyphs: Ruptures in Classical Cinema. Minneapolis: University of Minnesota Press, 1991.

Deleuze, Gilles. Le Pli. Leibniz et le baroque. Paris: Minuit, 1988.

Deleuze, Gilles. Foucault. Paris: Minuit, 1986.

Deleuze, Gilles. L'Image-temps. Paris: Minuit, 1985.

Deleuze, Gilles. L'Image-mouvement. Paris : Minuit, 1983.

Foucault, Michel. Surveiller et punir. Paris: Gallimard, 1975.

Gundersheimer, Werner (direction). The Dance of Death: 41 Woodcuts by Hans Holbein the Younger. New York: Dover, 1971.

Jacob, Christian. L'empire des cartes. Approche théorique de la cartographie à travers l'histoire. Paris: Albin Michel, 1992.

Kassovitz, Mathieu. Jusqüici tout va bien... Scénario et photographie autour du film $\mathrm{La}$ Haine. Montpellier: Actes Sud, 1995.

Metz, Christian. L'Énonciation impersonnelle ou le site du film. Paris: MéridiensKlincksieck, 1991.

Nuti, Lucia. «Le langage de la peinture dans la cartographie topographique», dans Catherine Bousquet-Bressolier (direction), L'CEil du cartographe et la représentation géographique du Moyen Âge à nos jours. Paris: CHTS (1995), p. 42-55.

Ropars-Wuilleumier, Marie-Claire. Le Texte divisé. Paris : P.U.F., 1981.

Wolff, Francis. Dire le monde. Paris: P.U.F., 1997. 